\title{
Triple negative apocrine breast carcinoma has better prognosis despite poor response to neoadjuvant chemotherapy
}

Taobo Hu

Peking University People's Hospital

Yiqiang Liu

Peking University Cancer Hospital

Xuejiao Lina Hu

Alaska Native Medical Center

Guiyang Zhao

Beijing Changping Hospital

\section{Shu Wang}

Peking University People's Hospital

Mengping Long ( $\square$ mplong@connect.ust.hk)

Peking University Cancer Hospital

\section{Research Article}

Keywords: Invasive apocrine carcinoma, Triple-negative breast cancer, Androgen receptor, Neoadjuvant therapy

Posted Date: January 12th, 2022

DOI: https://doi.org/10.21203/rs.3.rs-1242664/v1

License: (c) (1) This work is licensed under a Creative Commons Attribution 4.0 International License. Read Full License

Version of Record: A version of this preprint was published at Journal of Clinical Medicine on March 14th, 2022. See the published version at https://doi.org/10.3390/jcm11061607. 


\section{Abstract}

Background: Apocrine carcinoma is a rare subtype of invasive ductal breast cancer that shows apocrine differentiation and largely with triple negative immunohistology. Triple negative breast cancers are known to have a more aggressive clinical course. However, unlike the most other types, it is reported that triple negative apocrine carcinoma has a better prognosis. Due to scarcity of reported studies, our knowledges for its clinical behavior, prognosis and response to therapy are very limited.

Methods: In this study, we retrospectively retrieved 41 triple negative apocrine carcinoma cases from our breast cancer database with an average follow up 32.8 months.

Results: It was found that triple negative apocrine carcinoma had poorer response to neoadjuvant therapy, but better prognosis compared with other non-apocrine types of triple negative breast cancer. Meanwhile, triple negative apocrine carcinoma has a low proliferative nature as indicated by its low Ki67 index. Analysis of SEER database showed that chemotherapy did not improve breast cancer specific survival in TNAC patients.

Conclusions: Our results suggest that triple negative apocrine carcinoma is a special subtype of triple negative breast cancer for which de-escalation of chemotherapy should be considered.

\section{Background}

Apocrine carcinoma is a rare histologic subtype of breast cancer, accounting for about $1 \%$ of all breast cancers and is diagnosed by the apocrine differentiation of the cancer cells [1]. Apocrine carcinoma are often ER and PR negative, with $30 \%$ of them having HER2 amplification [2]. Thus, majority of the apocrine carcinoma is triple negative which means they lack the expression of ER, PR and HER2 and can be named as triple negative apocrine carcinoma (TNAC).

Although TNAC does not express ER and PR, another steroid hormone receptor androgen receptor (AR) is often positive in TNAC. AR was also expressed in $10-63 \%$ of triple negative breast cancer $[3,4]$ and the expression of AR is reported to be related with good prognosis in early breast cancer in terms of both disease-free survival and overall survival $[5,6]$. Triple negative breast cancer (TNBC) can be classified into a four-subtype system by various algorithms using transcriptomic data [7-10] and all the currently applied subtyping algorithms could distinguish one consistent molecular subtype which is the luminal androgen receptor (LAR) subtype . LAR accounted for $15-20 \%$ of all TNBC and is characterized by the high expression of the AR gene and enrichment in hormonally regulated pathways. LAR subtype is reported to be low proliferative and its distant metastasis often occur after 3 years [11]. About $59 \%$ of LAR showed histologic apocrine differentiation in more than $10 \%$ of all the cancer cells [12]. However, the exact correlation between TNAC and LAR subgroup in terms of molecular characteristics and clinical features is still largely unknown due to the limited cases of reported TNAC.

The analysis of both SEER database and National Cancer Database (NCD) showed that TNAC has a better prognosis than TNBC $[13,14]$. Study comparing TNAC that did not receive adjuvant chemotherapy with matched triple negative invasive ductal carcinoma that received adjuvant chemotherapy showed that the two groups share similar prognosis which indicate a potential de-escalation in the management of TNAC [15]. However, our understanding about the response of TNAC to neoadjuvant therapy is limited $[16,17]$. Nagao et al reported that in five invasive apocrine carcinoma that received neoadjuvant chemotherapy, none of them achieved pathological complete response (pCR) [17].

In this study, we compared the clinicopathological characteristics and survival of 41 TNAC cases with paired TNBC cases in which 21 of them have received neoadjuvant therapy and response was evaluated with the Miller-Payne grading system.

\section{Methods}

The study was approved by Peking University Cancer Hospital ethics committee (Reference number 2020KT113). The pathology database in Peking university Cancer hospital was queried for breast apocrine carcinomas diagnosed between 2008 and 2021 . A total of 41 cases who met diagnostic criteria including at least $95 \%$ of tumor showing apocrine differentiation, N:C ratio of tumor cells being 1:2 or more with abundant eosinophilic cytoplasm, prominent nucleoli and sharply defined cell borders were included and independently verified by two pathologists. Patients with only apocrine carcinoma in situ were excluded. These 41 patients 
also had tumor surgical resection data to achieve accuracy for pathological staging. ER, PR an HER2 status was evaluated by immunohistochemical staining and fluorescent in situ hybridization (FISH). Each of the TNAC cases has been paired with one nonapocrine triple-negative breast cancer (TNBC-NA) case by age at diagnosis, year of diagnosis and the receipt of neoadjuvant therapy to exclude the bias effect on prognosis. All of the paired TNBC-NA cases were invasive breast cancer of no special type. In the 41 cases of TNAC, 21 of them have received neoadjuvant therapy and were included in the neoadjuvant comparing group. The other 20 cases were included in the non-neoadjuvant group and all of them have received adjuvant chemotherapy. The survival was analyzed by Kaplan-Meier estimate and the response of 21 TNAC cases that received therapy was evaluated with the MillerPayne grading system. Ki-67 score is defined as the percentage of positively nuclear stained cells divided by the total number of malignant cells scored. When the staining is homogenous across sample, global Ki-67 score was used and for heterogenous staining Ki-67 score counted in hot spots region.

TNAC cases from SEER database were obtained from the SEER ${ }^{\star}$ Stat software, version 8.3.9.2. A total of 442 triple negative apocrine carcinoma patients were identified from 2010 to 2018 according to the following selection criteria: invasive apocrine carcinoma aged over 18 years old, negative ER and PR status, negative HER2 status, detailed information about survival was available. Propensity score matching (PSM) was used to achieve a 1:1 matching between chemotherapy and non-chemotherapy groups to reduce the compound effects caused by baseline information bias. R package "Matchlt" was employed for PSM [18].

\section{Results}

\section{Patients with TNAC have better prognosis than TNBC-NA despitea poorer response to neoadjuvant chemotherapy}

The immunohistochemical stain of protein markers including ER, PR, HER2, Ki-67, AR, EGFR and GCDFP-15, together with the hematoxylin and eosin stain in breast cancer tissues of TNAC and TNBC-NA groups was illustrated in Figure 1. Interestingly, in 11 cases of the TNAC group (11/41, 27\%), the HER2 immunostaining showed intense cytoplasmic granular signal which was reported as equivocal 2+ or 1+ (Figure 1). Whereas, further FISH performed all proved HER2 as non-amplified. Moreover, when another clone of HER2 antibody was applied for immunohistochemical staining, the cytoplasmic signal disappeared which indicated that the cytoplasmic signal could be non-specific (data not shown). The clinicopathologic characteristics of the 21 TNAC cases that received neoadjuvant therapy and their paired TNBC-NA case were summarized in Table 1 and that of the non-neoadjuvant therapy group were displayed in Table 2. The median age at diagnosis for TNAC group was 57 years (range from 36-77 years). Among them, 15 patients $(15 / 41,36.5 \%)$ has received lumpectomy and the other 26 patients $(26 / 41,63.5 \%)$ received mastectomy. The Ki67 positive percentage and the percentage of tumor infiltrating lymphocytes (TILs) was evaluated using the biopsy sample before any treatment. The mean Ki-67 positive percentage of TNAC was significantly lower than matched TNBC-NA in both neoadjuvant group and non-neoadjuvant group $(\mathrm{P}<0.05)$ (Figure 2). The histologic grade of TNAC group was less advanced compared with TNBC-NA group in the non-neoadjuvant group but was similar in the neoadjuvant group. For the percentage of stromal TILs, TNAC has a tendency of less TILs compared with TNBC-NA although it was not statistically significant in either non-neoadjuvant or neoadjuvant group. Other clinicopathological features including surgery type and radiation therapy showed no significant difference between TNAC and TNBC-NA.

For survival analysis, the median time of follow-up was 32.8 months (range 1.2-35.6 months). In TNAC group, none of the 21 patients died at the time of last follow-up while five patients in the TNBC-NA group $(5 / 41,12 \%)$ have died all due to breast cancer. The Overall survival (OS) of TNAC was better than TNBC-NA ( $\mathrm{P}=0.02)$ (Figure 2). For distant-metastasis free survival (DMFS) analysis, only one patient in TNAC group has experienced distant metastasis. Thus the DMFS survival curves of the two groups showed the same trend as in OS whereas the p-value was 0.052 which was marginally statistically significant possibly due to the small sample size. The disease-free survival (DFS), which includes local recurrence of the two groups, showed no significant difference (Figure 2).

Twenty-one cases in the TNAC group have received neoadjuvant therapy and pathological response was evaluated using MillerPayne grading system in the surgery sample which classified the response into 5 grades according to tumor cellularity in the lumpectomy/mastectomy specimen as compared with the pre-treatment biopsy [19]. In TNAC group, none of the 21 cases have achieved pathological complete response (pCR) which was grade 5 in Miller-Payne grading system while 4 patients (4/21, 19\%) in the TNBC-NA group has achieved pCR. Generally, the tumor response evaluated was much poorer in TNAC than that in matched 
TNBC-NA group ( $P=0.024)$ as displayed in Figure 3 . The detailed clinical information of the TNAC cases in the neoadjuvant group including neoadjuvant therapy regimen, clinical evaluation of response, tumor and lymph node stage, Ki67 positive percentage, histologic grade and TILs percentage were listed in Table 3.

\section{Chemotherapy did not improve breast cancer specific survival for TNAC patients}

Although TNAC showed poor response to neoadjuvant chemotherapy, whether patients with TNAC would benefit from chemotherapy was still unknown. Previous studies identified that TNAC was better than survival than TNBC by SEER database [20-22]. Further, Wu et a/ reported that in TNAC, patients with chemotherapy have better overall survival (OS) better than those without chemotherapy by SEER database [13]. However, their findings is limited by the absence of propensity score matching (PSM) and thus could be confounded by baseline bias. Also, the effect of chemotherapy on breast cancer specific survival (BCSS) of TNAC has not been elucidated. We next investigated the effect of chemotherapy on OS and BCSS of TNAC patients using SEER database with and without PSM. A total of 442 patients with TNAC were enrolled in the study which were divided into the chemo and no chemo groups according to whether they have received chemotherapy. There were 291 (65.8\%) patients in the chemo and 151 (34.2\%) patients in the no chemo group. The demographic and clinicopathological information of them were displayed and compared in Table 4. Patients in the chemo group were presented with younger age at diagnosis, more advanced stage status, higher histology grade than those in the chemo group. Also, patients in the chemo group were more likely to receive surgery and radiation therapy. To eliminate the bias in baseline information between the two groups, a 1:1 matching was performed using propensity score matching. After matching, 87 patients remained in both groups and there was no difference in demographic or

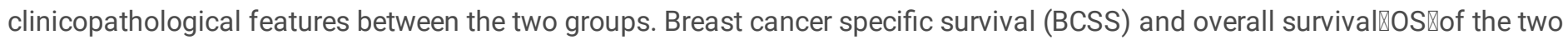
groups both before and after matching were plotted by Kaplan-Meire survial curve (Figure 4). Before matching, patients in chemo group showed better survival than no chemo group while there was no difference in BCSS between the two groups. For the two groups after PSM, the results were the same as in the before matching groups indicating that chemotherapy did not improve breast cancer specific survival for TNAC patients.

\section{Discussion}

Apocrine carcinoma is a special subtype of breast cancer characterized by apocrine metaplasia histologically and the activation of AR pathway molecularly. The mutational rate of PI3KCA gene in TNAC was $72 \%$, higher than other triple negative breast cancer including 55\% mutation rate in LAR group [23,24]. Despite all the difference, TNAC is treated the same with other triple negative breast cancer though the benefit of AR antagonist bicultamide was investigated in advanced ER and PR negative patients [25]. Our results suggest that TNAC is a low-risk group of TNBC that has low proliferation rate, better prognosis, and poor chemotherapy responsiveness. The pCR rate for LAR group was 10\% which was the lowest among the four molecular subtypes of TNBC [11]. While in our study, none of the 21 TNAC cases has achieved pCR under various treatment regimens, suggesting TNAC as a special subtype of AR-positive TNBC. Although in this study we have matched TNAC with TNBC-NA cases to reduce selection bias, our study is still limited by its retrospective nature. However, conducting a random clinical trial with a rare disease is also challenging.

Previous study showed that compared with luminal breast cancer, triple negative breast cancer has higher sensitivity to anthracycline-based neodjuvant chemotherapy but worse prognosis which could be contributed by the higher relapse rate in nonpCR patients [26]. In this study, TNAC showed the same pattern as luminal breast cancer which could be contributed by the expression of AR and other luminal genes in TNAC. AR-positive TNBC was known to have a favorable prognosis than AR-negative TNBC possibly contributed by the anti-proliferative effect of AR [27]. Meanwhile, anti-androgen receptor therapeutics including bicalutamide, enzalutamide and abiraterone have shown clinical benefit ratio ranging from $19 \%$ to $35 \%$ in AR-positive TNBC $[25,28,29]$.

\section{Conclusions}

Our results suggest that triple negative apocrine carcinoma is a special subtype of triple negative breast cancer which has better prognosis despite poor response to neoadjuvant chemotherapy. Thus, for the management of TNAC, it is necessary to conduct research evaluating the benefit of chemotherapy de-escalation and the adding of anti-androgen receptor therapy. 


\section{Abbreviations}

\begin{tabular}{ll} 
AR & Androgen receptor \\
\hline BCSS & Breast cancer specific survival \\
\hline DMFS & Distant metastasis free survival \\
\hline ER & Estrogen receptor \\
\hline OS & Overall survival \\
\hline pCR & Pathological complete response \\
\hline PR & Progesterone receptor \\
\hline PSM & Propensity score matching \\
\hline TNAC & Triple negative apocrine carcinoma \\
\hline TNBC & Triple negative breast cancer
\end{tabular}

\section{Declarations}

\section{Ethics approval and consent to participate}

The study was approved by Peking University Cancer Hospital ethics committee (Reference number 2020KT113). People who participated in this research had complete clinical data. Signed informed consents were obtained from the patients and/or the guardians.

\section{Consent for publication}

Informed consent has been obtained from the patients to use their samples for publication of the findings as per institutional ethics norms.

\section{Availability of data and materials}

All data generated or analyzed during this study are included in this published article.

\section{Competing interests}

None.

\section{Funding}

The author(s) disclosed receipt of the following financial support for the research, authorship, and/or publication of this article: This work was supported by the National Natural Science Foundation of China (Grant No. 82002979), Scientific Research and Development Funds of Peking University People's Hospital (Grant No. RDY2020-16) and the Young Investigator Program of Peking University Health Science Center (Grant No. BMU2020PYB022, BMU2021PYB013).

\section{Authors' contributions}

M.L. and T.H. performed study concept and design; M.L. , T.H. and X.H. performed writing and review of the paper; M.L. , T.H. and Y.L. provided acquisition, analysis and interpretation of data, and statistical analysis. All authors read and approved the final paper.

\section{Acknowledgements}

None.

\section{References}


1. Dellapasqua, S.; Maisonneuve, P.; Viale, G.; Pruneri, G.; Mazzarol, G.; Ghisini, R.; Mazza, M.; lorfida, M.; Rotmensz, N.; Veronesi, P.; et al. Immunohistochemically defined subtypes and outcome of apocrine breast cancer. Clin. Breast Cancer 2013, 13, 95-102, doi:10.1016/j.clbc.2012.11.004.

2. Vranic, S.; Marchiò, C.; Castellano, I.; Botta, C.; Scalzo, M.S.; Bender, R.P.; Payan-Gomez, C.; Di Cantogno, L.V.; Gugliotta, P.; Tondat, F.; et al. Immunohistochemical and molecular profiling of histologically defined apocrine carcinomas of the breast. Hum. Pathol. 2015, 46, 1350-1359, doi:10.1016/j.humpath.2015.05.017.

3. Astvatsaturyan, K.; Yue, Y.; Walts, A.E.; Bose, S. Androgen receptor positive triple negative breast cancer: Clinicopathologic, prognostic, and predictive features. PLoS One 2018, 13, e0197827, doi:10.1371/journal.pone.0197827.

4. Niemeier, L.A.; Dabbs, D.J.; Beriwal, S.; Striebel, J.M.; Bhargava, R. Androgen receptor in breast cancer: expression in estrogen receptor-positive tumors and in estrogen receptor-negative tumors with apocrine differentiation. Mod. Pathol. 2010, 23, 205-212, doi:10.1038/modpathol.2009.159.

5. Vera-Badillo, F.E.; Templeton, A.J.; Gouveia, P. de; Diaz-Padilla, I.; Bedard, P.L.; Al-Mubarak, M.; Seruga, B.; Tannock, I.F.; Ocana, A.; Amir, E. Androgen receptor expression and outcomes in early breast cancer: a systematic review and meta-analysis. J. Natl. Cancer Inst. 2014, 106, djt319, doi:10.1093/jnci/djt319.

6. Bozovic-Spasojevic, I.; Zardavas, D.; Brohée, S.; Ameye, L.; Fumagalli, D.; Ades, F.; Azambuja, E. de; Bareche, Y.; Piccart, M.; Paesmans, M.; et al. The Prognostic Role of Androgen Receptor in Patients with Early-Stage Breast Cancer: A Meta-analysis of Clinical and Gene Expression Data. Clin. Cancer Res. 2017, 23, 2702-2712, doi:10.1158/1078-0432.CCR-16-0979.

7. Burstein, M.D.; Tsimelzon, A.; Poage, G.M.; Covington, K.R.; Contreras, A.; Fuqua, S.A.W.; Savage, M.I.; Osborne, C.K.; Hilsenbeck, S.G.; Chang, J.C.; et al. Comprehensive genomic analysis identifies novel subtypes and targets of triple-negative breast cancer. Clin. Cancer Res. 2015, 21, 1688-1698, doi:10.1158/1078-0432.CCR-14-0432.

8. Jiang, Y.-Z.; Ma, D.; Suo, C.; Shi, J.; Xue, M.; Hu, X.; Xiao, Y.; Yu, K.-D.; Liu, Y.-R.; Yu, Y.; et al. Genomic and Transcriptomic Landscape of Triple-Negative Breast Cancers: Subtypes and Treatment Strategies. Cancer Cel/ 2019, 35, 428-440.e5, doi:10.1016/j.ccell.2019.02.001.

9. Lehmann, B.D.; Bauer, J.A.; Chen, X.; Sanders, M.E.; Chakravarthy, A.B.; Shyr, Y.; Pietenpol, J.A. Identification of human triplenegative breast cancer subtypes and preclinical models for selection of targeted therapies. J. Clin. Invest. 2011, 121, 2750-2767, doi:10.1172/JCl45014.

10. Lehmann, B.D.; Jovanović, B.; Chen, X.; Estrada, M.V.; Johnson, K.N.; Shyr, Y.; Moses, H.L.; Sanders, M.E.; Pietenpol, J.A. Refinement of Triple-Negative Breast Cancer Molecular Subtypes: Implications for Neoadjuvant Chemotherapy Selection. PLoS One 2016, 11, e0157368, doi:10.1371/journal.pone.0157368.

11. Masuda, H.; Baggerly, K.A.; Wang, Y.; Zhang, Y.; Gonzalez-Angulo, A.M.; Meric-Bernstam, F.; Valero, V.; Lehmann, B.D.; Pietenpol, J.A.; Hortobagyi, G.N.; et al. Differential response to neoadjuvant chemotherapy among 7 triple-negative breast cancer molecular subtypes. Clin. Cancer Res. 2013, 19, 5533-5540, doi:10.1158/1078-0432.CCR-13-0799.

12. Kim, S.; Moon, B.-I.; Lim, W.; Park, S.; Cho, M.S.; Sung, S.H. Feasibility of Classification of Triple Negative Breast Cancer by Immunohistochemical Surrogate Markers. Clin. Breast Cancer 2018, 18, e1123-e1132, doi:10.1016/j.clbc.2018.03.012.

13. Wu, W.; Wu, M.; Peng, G.; Shi, D.; Zhang, J. Prognosis in triple-negative apocrine carcinomas of the breast: A population-based study. Cancer Med. 2019, 8, 7523-7531, doi:10.1002/cam4.2634.

14. Arciero, C.A.; Diehl, A.H.; Liu, Y.; Sun, Q.; Gillespie, T.; Li, X.; Subhedar, P. Triple-negative apocrine carcinoma: A rare pathologic subtype with a better prognosis than other triple-negative breast cancers. J. Surg. Oncol. 2020, 122, 1232-1239, doi:10.1002/jso.26129. 
15. Montagna, E.; Cancello, G.; Pagan, E.; Bagnardi, V.; Munzone, E.; Dellapasqua, S.; Viale, G.; Mazzarol, G.; Veronesi, P.; Galimberti, V.; et al. Prognosis of selected triple negative apocrine breast cancer patients who did not receive adjuvant chemotherapy. Breast 2020, 53, 138-142, doi:10.1016/j.breast.2020.07.003.

16. Imamovic, D.; Bilalovic, N.; Skenderi, F.; Beslagic, V.; Ceric, T.; Hasanbegovic, B.; Beslija, S.; Vranic, S. A clinicopathologic study of invasive apocrine carcinoma of the breast: A single-center experience. Breast J. 2018, 24, 1105-1108, doi:10.1111/tbj.13140.

17. Nagao, T.; Kinoshita, T.; Hojo, T.; Tsuda, H.; Tamura, K.; Fujiwara, Y. The differences in the histological types of breast cancer and the response to neoadjuvant chemotherapy: the relationship between the outcome and the clinicopathological characteristics. Breast 2012, 21, 289-295, doi:10.1016/j.breast.2011.12.011.

18. Austin, P.C. An Introduction to Propensity Score Methods for Reducing the Effects of Confounding in Observational Studies. Multivariate Behav. Res. 2011, 46, 399-424, doi:10.1080/00273171.2011.568786.

19. Ogston, K.N.; Miller, I.D.; Payne, S.; Hutcheon, A.W.; Sarkar, T.K.; Smith, I.; Schofield, A.; Heys, S.D. A new histological grading system to assess response of breast cancers to primary chemotherapy: prognostic significance and survival. Breast 2003, 12, 320327, doi:10.1016/s0960-9776(03)00106-1.

20. Zhang, N.; Zhang, H.; Chen, T.; Yang, Q. Dose invasive apocrine adenocarcinoma has worse prognosis than invasive ductal carcinoma of breast: evidence from SEER database. Oncotarget 2017, 8, 24579-24592, doi:10.18632/oncotarget.15597.

21. Saridakis, A.; Berger, E.R.; Harigopal, M.; Park, T.; Horowitz, N.; Le Blanc, J.; Zanieski, G.; Chagpar, A.; Greenup, R.; Golshan, M.; et al. Apocrine Breast Cancer: Unique Features of a Predominantly Triple-Negative Breast Cancer. Ann. Surg. Oncol. 2021, 28, 56105616, doi:10.1245/s10434-021-10518-9.

22. Liao, H.-Y.; Zhang, W.-W.; Sun, J.-Y.; Li, F.-Y.; He, Z.-Y.; Wu, S.-G. The Clinicopathological Features and Survival Outcomes of Different Histological Subtypes in Triple-negative Breast Cancer. J. Cancer 2018, 9, 296-303, doi:10.7150/jca.22280.

23. Bareche, Y.; Venet, D.; Ignatiadis, M.; Aftimos, P.; Piccart, M.; Rothe, F.; Sotiriou, C. Unravelling triple-negative breast cancer molecular heterogeneity using an integrative multiomic analysis. Ann. Oncol. 2018, 29, 895-902, doi:10.1093/annonc/mdy024.

24. Sun, X.; Zuo, K.; Yao, Q.; Zhou, S.; Shui, R.; Xu, X.; Bi, R.; Yu, B.; Cheng, Y.; Tu, X.; et al. Invasive apocrine carcinoma of the breast: clinicopathologic features and comprehensive genomic profiling of 18 pure triple-negative apocrine carcinomas. Mod. Pathol. 2020, 33, 2473-2482, doi:10.1038/s41379-020-0589-x.

25. Gucalp, A.; Tolaney, S.; Isakoff, S.J.; Ingle, J.N.; Liu, M.C.; Carey, L.A.; Blackwell, K.; Rugo, H.; Nabell, L.; Forero, A.; et al. Phase II trial of bicalutamide in patients with androgen receptor-positive, estrogen receptor-negative metastatic Breast Cancer. Clin. Cancer Res. 2013, 19, 5505-5512, doi:10.1158/1078-0432.CCR-12-3327.

26. Carey, L.A.; Dees, E.C.; Sawyer, L.; Gatti, L.; Moore, D.T.; Collichio, F.; Ollila, D.W.; Sartor, C.I.; Graham, M.L.; Perou, C.M. The triple negative paradox: primary tumor chemosensitivity of breast cancer subtypes. Clin. Cancer Res. 2007, 13, 2329-2334, doi:10.1158/1078-0432.CCR-06-1109.

27. Wang, Y.; He, X.; Yu, Q.; Eng, C. Androgen receptor-induced tumor suppressor, KLLN, inhibits breast cancer growth and transcriptionally activates p53/p73-mediated apoptosis in breast carcinomas. Hum. Mol. Genet. 2013, 22, 2263-2272, doi:10.1093/hmg/ddt077.

28. Traina, T.A.; Miller, K.; Yardley, D.A.; Eakle, J.; Schwartzberg, L.S.; O'Shaughnessy, J.; Gradishar, W.; Schmid, P.; Winer, E.; Kelly, C.; et al. Enzalutamide for the Treatment of Androgen Receptor-Expressing Triple-Negative Breast Cancer. J. Clin. Oncol. 2018, 36, 884-890, doi:10.1200/JC0.2016.71.3495.

29. Bonnefoi, H.; Grellety, T.; Tredan, O.; Saghatchian, M.; Dalenc, F.; Mailliez, A.; L'Haridon, T.; Cottu, P.; Abadie-Lacourtoisie, S.; You, B.; et al. A phase II trial of abiraterone acetate plus prednisone in patients with triple-negative androgen receptor positive locally advanced or metastatic breast cancer (UCBG 12-1). Ann. Oncol. 2016, 27, 812-818, doi:10.1093/annonc/mdw067.

Page $7 / 18$ 


\section{Tables}

Table 1. Clinicopathological features of TNAC and TNBC-NA patients received no neoadjuvant therapy 


\begin{tabular}{|c|c|c|c|}
\hline & TNAC & TNBC-NA & $P$ \\
\hline Age at diagnosis (y) & & & 1.000 \\
\hline $20-49$ & $6(30.0)$ & $6(30.0)$ & \\
\hline $50-69$ & $11(55.0)$ & $11(55.0)$ & \\
\hline $70-89$ & $3(15.0)$ & $3(15.0)$ & \\
\hline T stage & & & 0.196 \\
\hline T1 & $16(80.0)$ & $11(55.0)$ & \\
\hline T2 & $4(20.0)$ & $8(40.0)$ & \\
\hline T3 & $0(0)$ & $0(0)$ & \\
\hline T4 & $0(0)$ & $1(5.0)$ & \\
\hline \multicolumn{4}{|l|}{$\mathrm{N}$ stage } \\
\hline NO & $18(90.0)$ & $14(70.0)$ & 0.348 \\
\hline $\mathrm{N} 1$ & $1(5.0)$ & $4(20.0)$ & \\
\hline N2 & & $1(5.0)$ & \\
\hline N3 & $1(5.0)$ & $1(5.0)$ & \\
\hline AJCC stage & & & 0.323 \\
\hline IA & $14(70.0)$ & $10(50.0)$ & \\
\hline IIA & $5(25.0)$ & $5(25.0)$ & \\
\hline IIB & $0(0)$ & $3(15.0)$ & \\
\hline IIIA & $0(0)$ & $1(5.0)$ & \\
\hline IIIB & $0(0)$ & $0(0)$ & \\
\hline IIIC & $1(5.0)$ & $1(5.0)$ & \\
\hline Radiation & & & 0.500 \\
\hline No & $15(75.0)$ & $12(60.0)$ & \\
\hline Yes & $5(25.0)$ & $8(40.0)$ & \\
\hline Surgery type & & & 1.000 \\
\hline BCS* & $7(35.0)$ & $6(30.0)$ & \\
\hline Mastectomy & $13(65.0)$ & $14(70.0)$ & \\
\hline Laterility & & & 0.747 \\
\hline Left & $7(35.0)$ & $9(45.0)$ & \\
\hline Right & $13(65.0)$ & $11(55.0)$ & \\
\hline Ki67 (\%) & & & $<0.001$ \\
\hline $0-29$ & $18(90.0)$ & $2(10.0)$ & \\
\hline $30-59$ & $2(10.0)$ & $3(15.0)$ & \\
\hline $60-99$ & $0(0.0)$ & $15(75.0)$ & \\
\hline Histologic grade & & & 0.006 \\
\hline
\end{tabular}




\begin{tabular}{llll} 
I & $8(40.0)$ & $1(5.0)$ & \\
II & $5(25.0)$ & $4(20.0)$ & \\
\hline III & $5(25.0)$ & $15(75.0)$ & \\
\hline Missing & $2(10.0)$ & & \\
\hline TILS & & & 0.051 \\
\hline $0-10$ & $11(61.1)$ & $6(30.0)$ & \\
\hline $11-40$ & $6(33.3)$ & $7(35.0)$ & \\
\hline $41-90$ & $1(5.6)$ & $7(35.0)$ &
\end{tabular}

BCS: breast conserving surgery

Table 2. Clinicopathological features of TNAC and TNBC-NA patients received neoadjuvant therapy 
TNAC TNBC-NA $P$

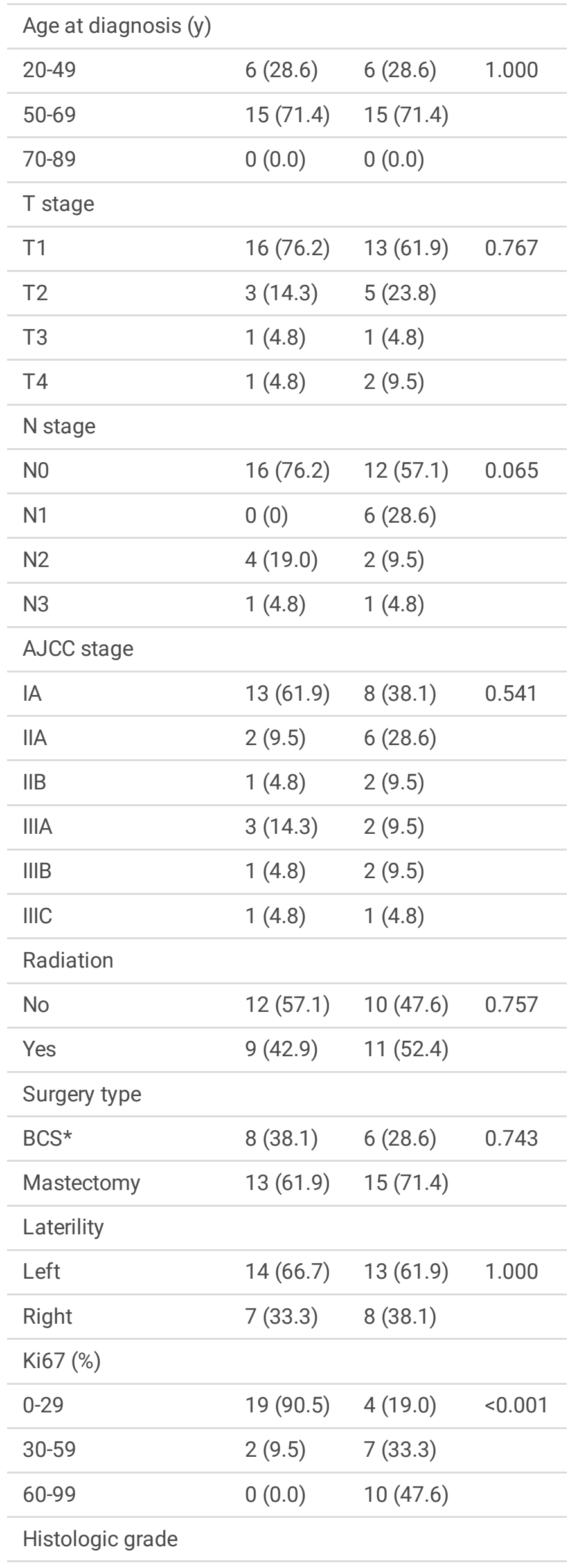




\begin{tabular}{llll} 
I & $6(28.6)$ & $2(9.5)$ & 0.164 \\
\hline II & $11(52.4)$ & $11(52.4)$ & \\
\hline III & $2(9.5)$ & $7(33.3)$ & \\
\hline Missing & $2(9.5)$ & $1(4.8)$ & \\
\hline TILs & & & \\
\hline $0-10$ & $14(73.7)$ & $9(45.0)$ & 0.097 \\
\hline $11-40$ & $2(10.5)$ & $8(40.0)$ & \\
\hline $41-90$ & $3(15.8)$ & $3(15.0)$ &
\end{tabular}

Table 3. Clinical information of TNAC patients who received neoadjuvant therapy 


\begin{tabular}{|c|c|c|c|c|c|c|c|c|}
\hline & $\begin{array}{l}\text { Neoadjuvant } \\
\text { therapy }\end{array}$ & Clinical evaluation & $\begin{array}{l}\mathrm{T} \\
\text { stage }\end{array}$ & $\begin{array}{l}\mathrm{N} \\
\text { stage }\end{array}$ & $\begin{array}{l}\text { MP } \\
\text { grade }\end{array}$ & Ki67(\%) & $\begin{array}{l}\text { Histologic } \\
\text { grade }\end{array}$ & TILs(\%) \\
\hline $\begin{array}{l}\text { TNAC- } \\
1\end{array}$ & ddEC/T1w & Unknown/SD & 1 & 0 & 3 & 5 & I & 2 \\
\hline $\begin{array}{l}\text { TNAC- } \\
2\end{array}$ & $\mathrm{AC} / \mathrm{T}$ & Unknown & 4 & 2 & 2 & 30 & II & 15 \\
\hline $\begin{array}{l}\text { TNAC- } \\
3\end{array}$ & TP1w/CEF/DF & $\mathrm{SD} / \mathrm{SD} / \mathrm{PR}$ & 1 & 0 & 2 & 20 & I & 5 \\
\hline $\begin{array}{l}\text { TNAC- } \\
4\end{array}$ & TP/CEF/NP & $\mathrm{SD} / \mathrm{SD} / \mathrm{PR}$ & 1 & 2 & 2 & 10 & II & 40 \\
\hline $\begin{array}{l}\text { TNAC- } \\
5\end{array}$ & TPX & PR & 1 & 0 & 2 & 5 & II & 8 \\
\hline $\begin{array}{l}\text { TNAC- } \\
6\end{array}$ & TP1w & PR & 1 & 0 & 4 & 10 & II & 1 \\
\hline $\begin{array}{l}\text { TNAC- } \\
7\end{array}$ & $\mathrm{ddEC} / \mathrm{T} 1 \mathrm{w}$ & $\mathrm{SD} / \mathrm{PR}$ & 1 & 0 & 3 & 20 & II & 1 \\
\hline $\begin{array}{l}\text { TNAC- } \\
8\end{array}$ & TP1w & SD & 1 & 0 & 3 & 10 & II & 60 \\
\hline $\begin{array}{l}\text { TNAC- } \\
9\end{array}$ & $\mathrm{CEF} / \mathrm{TP} 1 \mathrm{w}$ & Unknown/PR & 1 & 0 & 3 & 10 & I & 3 \\
\hline $\begin{array}{l}\text { TNAC- } \\
10\end{array}$ & $\mathrm{~T} 1 \mathrm{w} / \mathrm{EC}$ & Unknown/PR & 2 & 0 & 4 & 5 & II & 5 \\
\hline $\begin{array}{l}\text { TNAC- } \\
11\end{array}$ & $\mathrm{~T} 1 \mathrm{w} / \mathrm{AC}$ & Unknown/SD & 2 & 2 & 2 & 15 & II & 10 \\
\hline $\begin{array}{l}\text { TNAC- } \\
12\end{array}$ & ddEC/ddT175 & Unknown & 1 & 0 & 2 & 20 & I & 5 \\
\hline $\begin{array}{l}\text { TNAC- } \\
13\end{array}$ & $\mathrm{TP} 1 \mathrm{w} / \mathrm{X} / \mathrm{AC}$ & Unknown/PR & 1 & 0 & 3 & 15 & I & 0 \\
\hline $\begin{array}{l}\text { TNAC- } \\
14\end{array}$ & CEF & SD & 1 & 2 & 2 & 20 & Unknown & Unknown \\
\hline $\begin{array}{l}\text { TNAC- } \\
15\end{array}$ & $\mathrm{EC}$ & SD & 1 & 0 & 2 & 20 & II & 3 \\
\hline $\begin{array}{l}\text { TNAC- } \\
16\end{array}$ & TP/NE/DCF/NP & Unknown/Unknown/Unknown/PR & 1 & 0 & 2 & 5 & I & 0 \\
\hline $\begin{array}{l}\text { TNAC- } \\
17\end{array}$ & $\mathrm{CEF} / \mathrm{TP} 1 \mathrm{w}$ & Unknown/PR & 1 & 3 & 2 & 25 & III & 3 \\
\hline $\begin{array}{l}\text { TNAC- } \\
18\end{array}$ & ddEC/ddT175 & Unknown/PR & 1 & 0 & 3 & 20 & II & 45 \\
\hline $\begin{array}{l}\text { TNAC- } \\
19\end{array}$ & $\mathrm{ddEC} / \mathrm{T} 1 \mathrm{w}$ & Unknown/SD & 2 & 0 & 3 & 5 & II & 80 \\
\hline $\begin{array}{l}\text { TNAC- } \\
20\end{array}$ & $\mathrm{ddEC} / \mathrm{T} 1 \mathrm{w}$ & $\mathrm{PR} / \mathrm{SD}$ & 1 & 0 & 3 & 40 & Unknown & Unknown \\
\hline $\begin{array}{l}\text { TNAC- } \\
21\end{array}$ & $\mathrm{X} / \mathrm{T}$ & SD & 3 & 0 & 1 & 15 & III & 2 \\
\hline
\end{tabular}

Chemotherapy regimen abbreviation: EC- epirubicin and cyclophosphamide; T- docetaxel; AC- doxorubicin and cyclophosphamide; CEF- cyclophosphamide, epirubicin and fluorouracil; DF- daunorubicin and fluorouracil; TP- paclitaxel and platin; NP- vinorelbine and cisplatin; TPX-paclitaxel, platin and xeloda ;X-xeloda

Table 4. Baseline information of triple negative apocrine carcinoma patients from SEER database 


\begin{tabular}{|c|c|c|c|c|c|c|}
\hline & \multicolumn{3}{|l|}{ Before PSM } & \multicolumn{3}{|l|}{ After PSM } \\
\hline & No Chemo & Chemo & P-value & No Chemo & Chemo & P-value \\
\hline Sample size & 151 & 291 & & 87 & 87 & \\
\hline AgeGroup & & & $<0.001$ & & & 1.000 \\
\hline$<50$ years & $5(3.3)$ & 40 (13.7) & & $3(3.4)$ & $3(3.4)$ & \\
\hline $50-69$ years & $41(27.2)$ & $191(65.6)$ & & $36(41.4)$ & $36(41.4)$ & \\
\hline $70+$ years & $105(69.5)$ & $60(20.6)$ & & $48(55.2)$ & $48(55.2)$ & \\
\hline Income & & & 0.579 & & & 0.456 \\
\hline$\$ 50,000-\$ 69,999$ & $74(49.0)$ & $129(44.3)$ & & $43(49.4)$ & $35(40.2)$ & \\
\hline$\$ 70,000+$ & $56(37.1)$ & $113(38.8)$ & & $30(34.5)$ & $34(39.1)$ & \\
\hline$<\$ 50,000$ & $21(13.9)$ & $49(16.8)$ & & $14(16.1)$ & $18(20.7)$ & \\
\hline Race & & & 0.542 & & & 0.765 \\
\hline Hispanic (All Races) & $11(7.3)$ & $25(8.6)$ & & $7(8.0)$ & $6(6.9)$ & \\
\hline Non-Hispanic Asian or Pacific Islander & $19(12.6)$ & $37(12.7)$ & & $13(14.9)$ & $16(18.4)$ & \\
\hline Non-Hispanic Black & $26(17.2)$ & $40(13.7)$ & & $13(14.9)$ & $9(10.3)$ & \\
\hline Non-Hispanic White & $95(62.9)$ & $185(63.6)$ & & $54(62.1)$ & $56(64.4)$ & \\
\hline Others & $0(0.0)$ & $4(1.4)$ & & $0(0.0)$ & $0(0.0)$ & \\
\hline Stage & & & $<0.001$ & & & 1.000 \\
\hline I & $81(53.6)$ & $68(23.4)$ & & $44(50.6)$ & $44(50.6)$ & \\
\hline II & $25(16.6)$ & $92(31.6)$ & & $18(20.7)$ & $18(20.7)$ & \\
\hline III & $5(3.3)$ & $27(9.3)$ & & $3(3.4)$ & $3(3.4)$ & \\
\hline IV & $1(0.7)$ & $5(1.7)$ & & $0(0.0)$ & $0(0.0)$ & \\
\hline Unknown & $39(25.8)$ & $99(34.0)$ & & $22(25.3)$ & $22(25.3)$ & \\
\hline Grade & & & $<0.001$ & & & 1.000 \\
\hline Well differentiated; Grade I & $21(13.9)$ & $12(4.1)$ & & $8(9.2)$ & $8(9.2)$ & \\
\hline Moderately differentiated; Grade II & $90(59.6)$ & $159(54.6)$ & & $62(71.3)$ & $62(71.3)$ & \\
\hline Poorly differentiated; Grade III & $34(22.5)$ & $106(36.4)$ & & $16(18.4)$ & $16(18.4)$ & \\
\hline Undifferentiated; anaplastic; Grade IV & $1(0.7)$ & $0(0.0)$ & & $0(0.0)$ & $0(0.0)$ & \\
\hline Unknown & $5(3.3)$ & $14(4.8)$ & & $1(1.1)$ & $1(1.1)$ & \\
\hline Sequence & & & 0.926 & & & 0.316 \\
\hline 1 st of 2 or more primaries & $19(12.6)$ & $39(13.4)$ & & $12(13.8)$ & $18(20.7)$ & \\
\hline One primary only & $132(87.4)$ & $252(86.6)$ & & $75(86.2)$ & $69(79.3)$ & \\
\hline Surgery & & & 0.033 & & & 1.000 \\
\hline No & $9(6.0)$ & $5(1.7)$ & & $0(0.0)$ & $0(0.0)$ & \\
\hline Yes & $142(94.0)$ & $286(98.3)$ & & 87 & 87 & \\
\hline Radiation & & & 0.019 & & & 1.000 \\
\hline
\end{tabular}




$\begin{array}{lllll}\text { No } & 77(51.0) & 113(38.8) & 39(44.8) & 39(44.8) \\ \text { Yes } & 74(49.0) & 178(61.2) & 48(55.2) & 48(55.2)\end{array}$

Figures
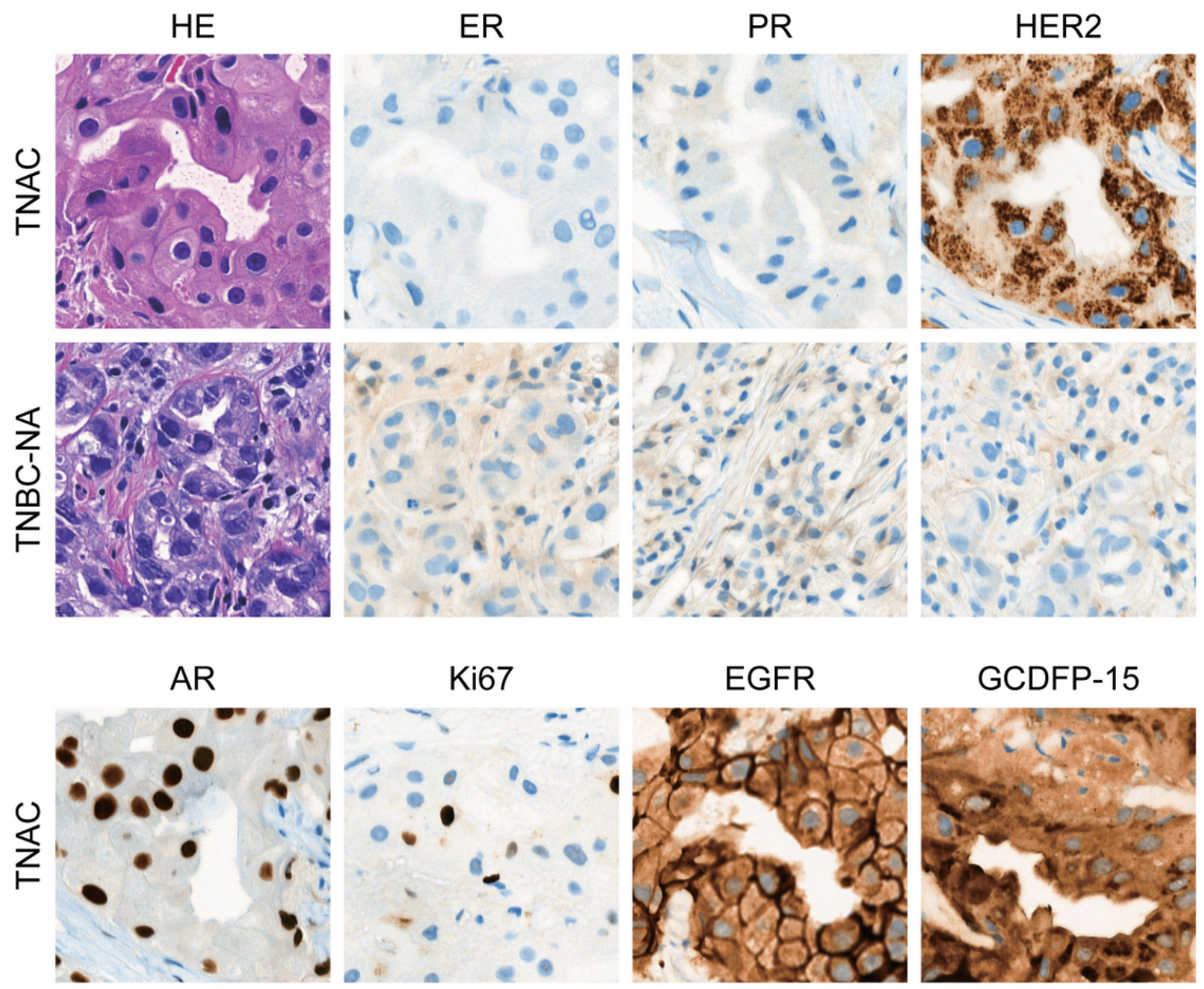

GCDFP-15

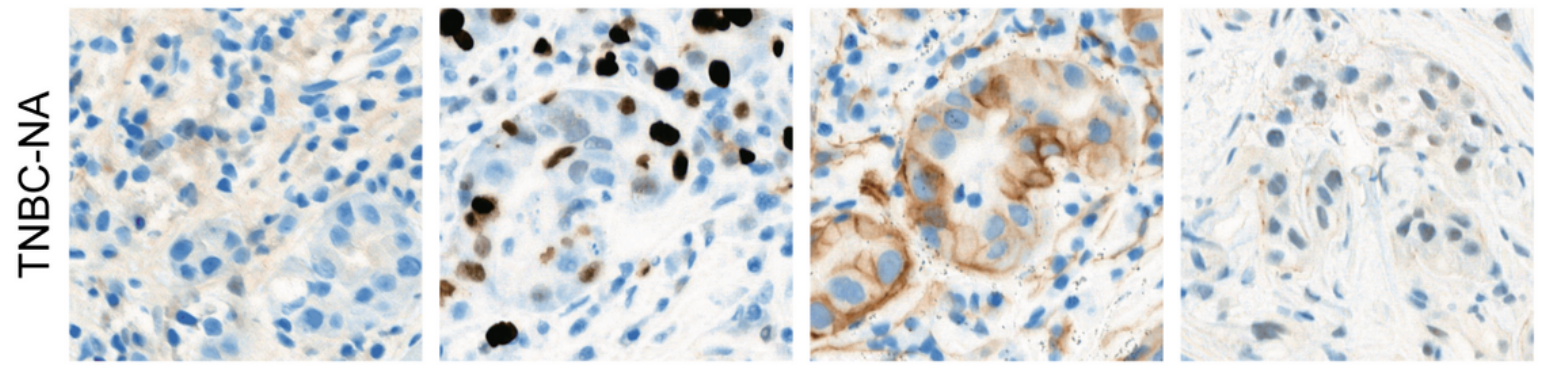

Figure 1

Representative HE and IHC staining images from TNAC and TNBC-NA patients.. 


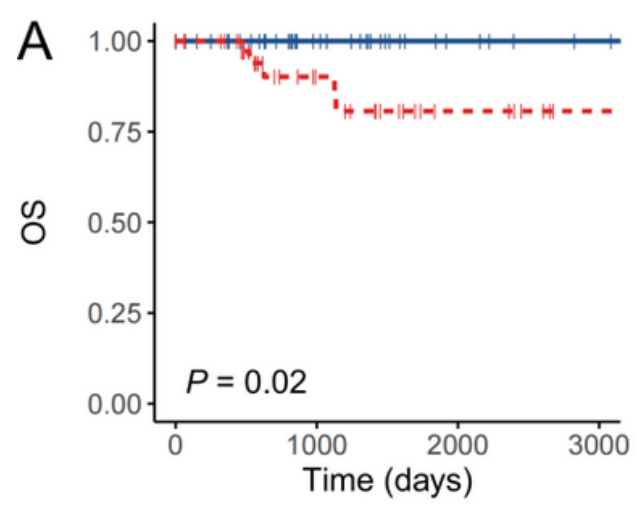

Number at risk

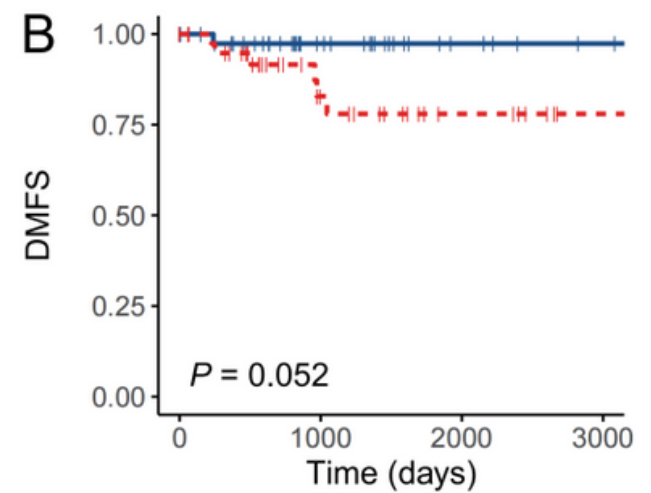

Number at risk

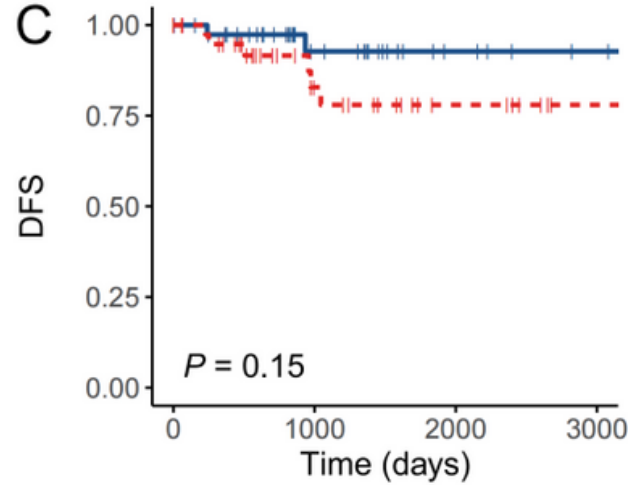

Number at risk

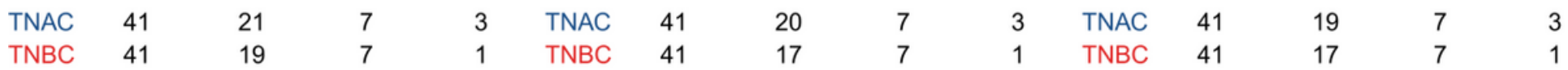

Figure 2

Survival plot of TNAC and TNBC-NA Cohort. Overall survival (OS), distant metastasis-free survival (DMSF) and disease-free survival (DFS) of TNAC and TNBC-NA groups were analyzed with Kaplan-Meier curve. 


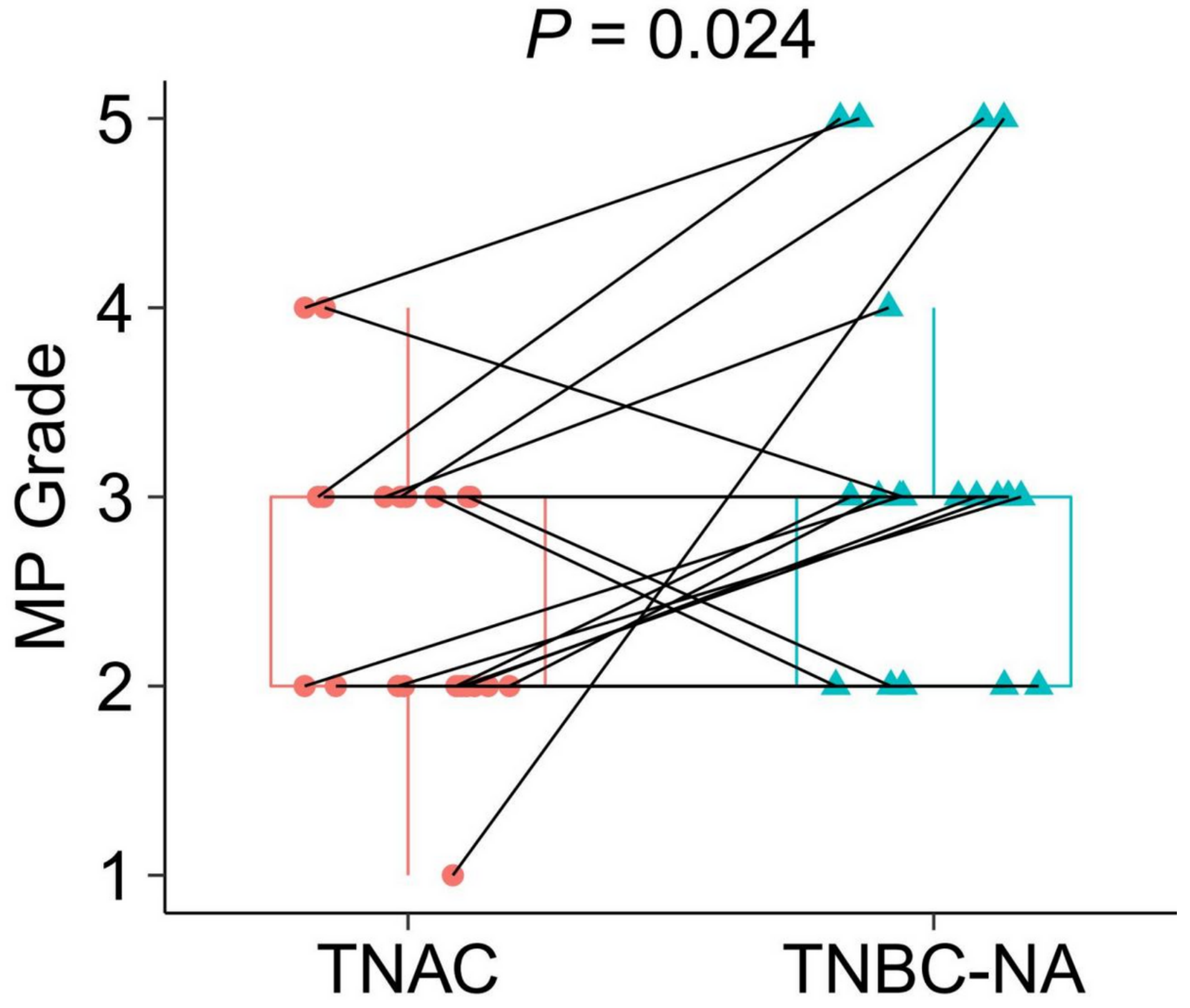

Figure 3

Paired Miller-Payne grading of study cohort. The Miller-Payne grade which represents neoadjuvant chemotherapy responsiveness was plotted with red dot (TNAC) and green triangle (TNBC-NA). Each TNAC and TNBC-NA pair was connected with a black line. Statistical analysis was performed with paired student's t-test. 


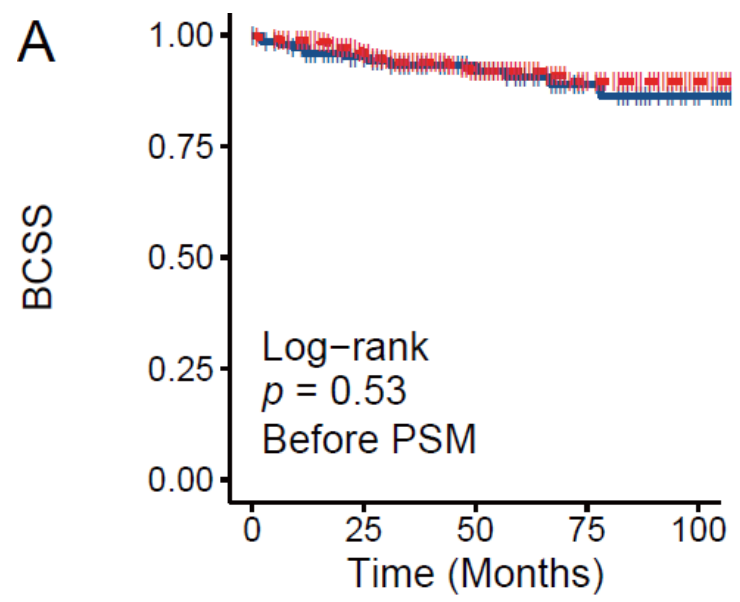

Number at risk

$\begin{array}{llllll}\text { No Chemo } & 151 & 112 & 73 & 38 & 12 \\ & 291 & 205 & 122 & 69 & 20\end{array}$

C

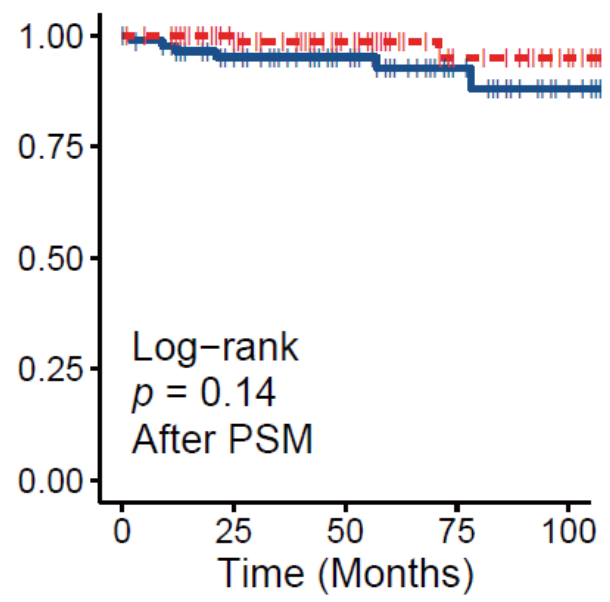

Number at risk

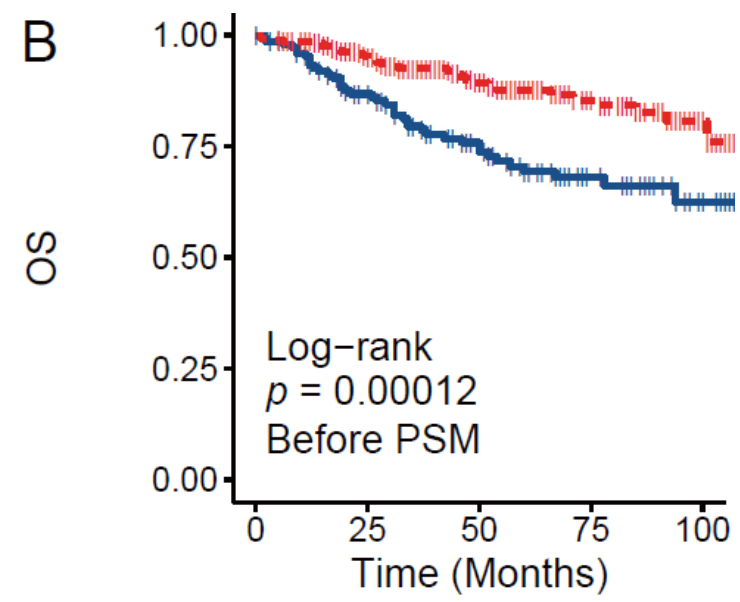

Number at risk

$\begin{array}{llllll}\text { No Chemo } & 151 & 112 & 73 & 38 & 12 \\ \text { Chemo } & 291 & 205 & 122 & 69 & 20\end{array}$

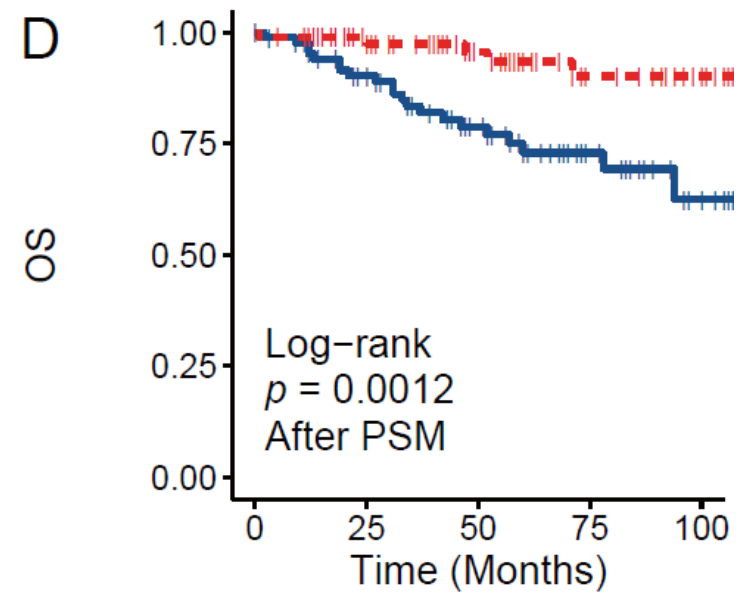

Number at risk

$\begin{array}{llllllllllll}\text { No Chemo } & 87 & 69 & 45 & 21 & 7 & \text { No Chemo } & 87 & 69 & 45 & 21 & 7 \\ \text { Chemo } & 87 & 70 & 46 & 23 & 8 & \text { Chemo } & 87 & 70 & 46 & 23 & 8\end{array}$

Figure 4

Survival plot of chemo group and no chemo group in TNAC patients from SEER. Breast cancer specific survival (BCSS) and overall survival (OS) of TNAC patients before matching ( $A$ and $B$ ) and after matching ( $C$ and $D)$ were analyzed with Kaplan-Meier curve. Number at risk was displayed below. 